\title{
Accurate coupling coefficients for fiber couplers with weakly fused cross sections
}

\author{
Hung-chun Chang, Ting-Huei Lin, and Tzong-Lin Wu
}

\begin{abstract}
For fused tapered fiber couplers with weakly fused cross sections, power coupling takes place between two touching tapered cladding cylinders embedded in the air, and the cylinders then become a strongly guiding structure. We provide accurate polarization-dependent coupling coefficients for such a structure, on the basis of a rigorous vectorial numerical calculation. Our results for the corresponding weakly guiding structure are compared with previous vector perturbation methods base on scalar fields. It is found that the scalar theories are not applicable to those cases with large normalized frequencies or with small fiber separations when the polarization-splitting effect is concerned.
\end{abstract}

The fused tapered biconical optical fiber coupler has become the key type of fiber coupler because of its low loss, better thermal stability, and ease of fabrication. ${ }^{1,2}$ When appropriately designed, the fused single-modefiber coupler can also be used for polarization beam splitting. ${ }^{3,4}$ Unlike the polished-type coupler, in which the structure remains weakly guiding, ${ }^{5}$ the light is guided in the neck region of the tapered coupler by the boundary between the fiber cladding and the external medium, which is air unless some other potting material is used. ${ }^{6}$ The fiber cores are reduced significantly and can be neglected in the coupling. It is well known that power coupling occurs through the spatial beating of the symmetric (even) and the antisymmetric (odd) modes on the composite waveguide of the neck region. The distance over which the power transfer becomes maximum, that is, the coupling length, is determined by the coupling coefficient, which is defined as half the difference between the propagation constants of the symmetric and the antisymmetric modes.

In practice a fused coupler can be either well (strongly) fused or weakly fused. ${ }^{4}$ The strongly fused coupler has a dumbbell shape in its cross section in the neck region, whereas for the weakly fused coupler, the coupling region is better modeled by two

The authors are with the Department of Electrical Engineering, National Taiwan University, Taipei, Taiwan 106-17, China. H.-C. Chang is also with the Graduate Institute of Electro-Optical Engineering, National Taiwan University.

Received 2 August 1994; revised manuscript received 7 April 1995.

0003-6935/95/276168-04\$06.00/0.

() 1995 Optical Society of America. touching circular cylinders. ${ }^{4}$ The individual cylinders are obviously strongly guiding fibers if the external region is air. Although analytical formulas for the coupling coefficient and its polarization corrections have been given for weakly guiding fibers, ${ }^{7,8}$ no such formulas exist for the strongly guiding structure. Therefore the weakly guiding results are sometimes taken as an approximation in the analysis of practical couplers. $^{2}$ Recently, Renner ${ }^{9}$ proposed a perturbation technique to derive the scalar first-order field corrections for the supermodes of couplers composed of two identical weakly guiding step-index fiber cores. He gave polarization corrections for the coupling coefficient with an accuracy to the lowest order of both weak coupling and the refractive index and pointed out that earlier results reported in Refs. 7 and 8 would be erroneous. We have commented on that error in an earlier paper. ${ }^{10}$

In this paper we provide accurate values for the coupling strength and for its polarization dependences by rigorously solving the (vectorial) boundaryvalue problem of two touching fibers. The solution is based on a circular-harmonics expansion method, and related results have been reported previously. ${ }^{11}$ Here we emphasize a structure with a large normalized frequency value and discuss the polarizationsplitting effect in the coupling, aiming at application to weakly fused couplers. Both the weakly guiding and the strongly guiding cases are studied, and the accuracy of the analytical formulas for the weakly guiding structure given in Refs. 8 and 9 is examined. Correcting coupling coefficients as given in this paper should be valuable in the study and design of various characteristics of fused couplers.

The cross section of the touching cylinder structure 


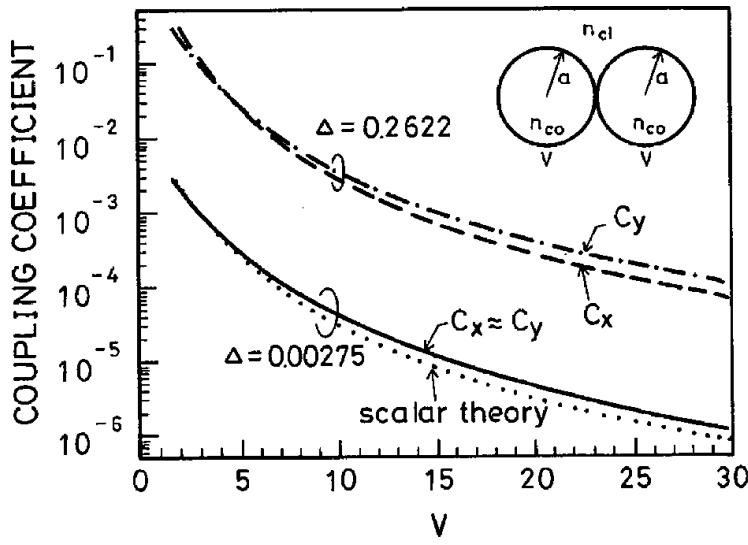

Fig. 1. Coupling coefficient versus normalized frequency $V$ for the structure consisting of two touching fibers. The dotted curve is obtained with the theory in Ref. 8.

is shown as an inset in Fig. 1, where $a$ is the core radius; $n_{\mathrm{co}}$ and $n_{\mathrm{cl}}$ denoted the indices of refraction of the core and cladding, respectively; and $V$ is the normalized frequency, defined as $V=2 \pi(a / \lambda)$ $\left(n_{\mathrm{co}}^{2}-n_{\mathrm{cl}}\right)^{2 / 2}$, with $\lambda$ being the light wavelength. When the external medium of the fused couplers is air $\left(n_{\mathrm{cl}}=1\right)$ and the original cladding is reduced to such an extent that $a=5 \mu \mathrm{m}$, we have $V \approx 25$ when $n_{\mathrm{co}}=$ 1.45 and $\lambda=1.3 \mu \mathrm{m}$ are used. Thus, in Figs. 1 and 2 we present the results up to $V=30$.

The symmetric and antisymmetric modes (supermodes) on the touching cylinder structure are solved through the use of the circular-harmonics expansion method proposed by Wijngaard. ${ }^{12}$ In the method the electromagnetic fields are expanded in terms of circular harmonics. Matching the continuity conditions at the core-cladding boundaries leads to a system of homogeneous equations, which yields the field properties of the vector modes when numerically solved. The full vectorial nature of the modes is considered in the solution, and thus the polarization effect in the

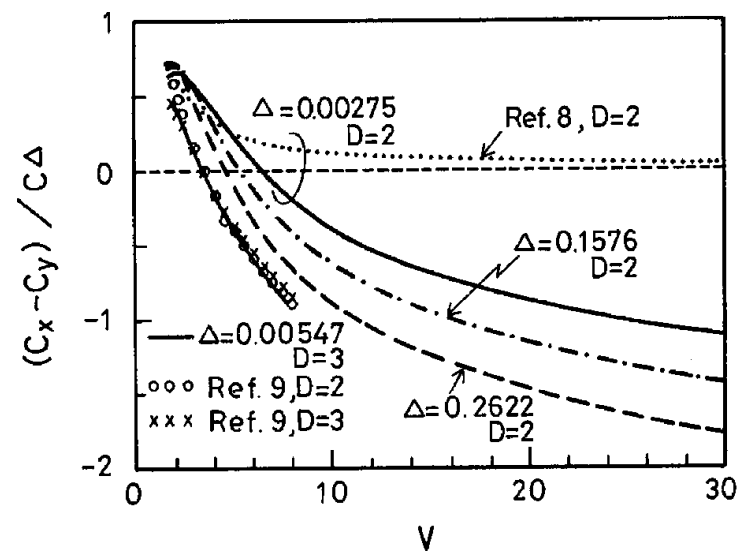

Fig. 2. Polarization effect versus normalized frequency $V$ for different $\Delta$ values and for core separation $D=2$ and 3 , as predicted by different theories. The dotted curve and the circles represent the scalar theory results of Refs. 8 and 9 , respectively, for $D=2$ and $\Delta=0.00275$. The X's represent the scalar theory results of Ref. 9 for $D=3$ and $\Delta=0.00547$. The other four curves represent our results based on the circular-harmonics expansion method. structure can be accurately examined. The coupling coefficient is calculated as half the difference between the obtained symmetric and antisymmetric supermode propagation constants. We denote the coupling coefficient as $C_{x}$ if the polarization of the corresponding two modes is predominantly in the $x$ (horizontal, referring to the inset in Fig. 1) direction and as $C_{y}$ if the polarization is predominantly in the $y$ (vertical) direction.

Figure 1 shows the coupling coefficient versus $V$ results for two cases, one in which the individual cylinders are weakly guiding (where $\Delta=0.00275$, $\left.n_{\mathrm{co}}=1.454, n_{\mathrm{cl}}=1.45\right)$ and the other in which the cylinders are strongly guiding $\left(\Delta=0.2622, n_{\text {co }}=1.45\right.$, $\left.n_{\mathrm{cl}}=1\right)$, where $\Delta=\left(1-n_{\mathrm{cl}}^{2} / n_{\mathrm{co}}^{2}\right) / 2$. For the weakly guiding case, for which $C_{x} \approx C_{y}$, the result of our rigorous numerical calculation is plotted as a solid curve, and that obtained with the analytical formula derived from the scalar theory without any vector correction $^{8}$ is shown as a dotted curve. The scalar result deviates from our rigorous calculation when $V$ is large. Note that the scalar coupling coefficient obtained in Ref. 9 is identical to that found in Ref. 8. For the strongly guiding case shown in Fig. 1, the difference between $C_{x}$ and $C_{y}$ is clearly demonstrated. One may notice that $C_{x}>C_{y}$ for small $V$ and $C_{y}$ becomes greater than $C_{x}$ for $V>5$.

For modeling the polarization-beam-splitting effect, we need the difference between $C_{x}$ and $C_{y}$. In Fig. 2 we plot $\left(C_{x}-C_{y}\right) / C \Delta$ as a function of $V$ for different $\Delta$ values. The quantity $C$ is taken as the average of $C_{x}$ and $C_{y}$. Except for the touching-core case $(D=2$, where $D$ is the normalized core separation and is defined as the ratio of the distance between the two core centers and the core radius $a$ ), some results for $D=3$ are also included. We discuss the results for $D=2$ first. The dotted curve represents the result obtained with the polarization-correction formula in Ref. 8. The calculation of Ref. 9 is included as circles in Fig. 2. They both show significant deviation from our rigorous calculation (solid curve) even for small $V$ values. For $D=2, C_{x}-C_{y}$ changes sign at a certain $V$ value for all three $\Delta$ values, whereas $C_{x}$ is always greater than $C_{y}$ if calculated according to Ref. 8. Our calculation reveals that for the weakly guiding case $(\Delta=0.00275)$, $C_{x}=C_{y}$ at $V \approx 6.3$, whereas the theory of Ref. 9 predicts that $C_{x}-C_{y}$ changes sign at $V \approx 3.454$. When $D=3$, our calculation for the weakly guiding case $(\Delta=0.00547)$, shown as the thinner solid curve linking the shapes in Fig. 2, is quite close to the prediction given in Ref. 9, shown as X's, for the range of $V$ values considered. Both of them cross the zero-value line at $V \approx 3.454$. The $\Delta$ value was increased in this calculation because for greater core separation it becomes difficult to solve the waveguide problem for small $\Delta$ values with the present method. The theory of Ref. 9 predicts that $C_{x}=C_{y}$ at $V \approx$ 3.454 for any separation $D$. One examination of the difference between the scalar perturbation theory and the exact calculation is made simply to compare 
their prediction of the $V$ value of the isotropic point $\left(C_{x}=C_{y}\right)$. The results obtained with our rigorous calculation for the $V$ values at which $C_{x}=C_{y}$ occurs for different core separations $D$ with $\Delta=0.00547$ are shown in Fig. 3. It appears that the $V$ value approaches 3.454 as $D$ increases. However, when moving toward the touching-core case, the scalar firstorder correction of Ref. 9 quickly loses its accuracy in providing accurate polarization-splitting quantities.

One thing worth noting is that our analysis results from Fig. 2 tell us that the polarization birefringence is still significant for couplers with large $V$ values when the two fibers are touching $(D=2)$. Previous study of the fused couplers by Zheng ${ }^{13}$ with a finiteelement analysis under the assumption that $V \rightarrow \infty$ predicted that the value of $C_{x}-C_{y}$ becomes zero when the fibers are nearly touching, or that slightly fused couplers are polarization insensitive. We have recently performed a rigorous analysis of form birefringence of fused couplers, ${ }^{14,15}$ showing that significant polarization birefringence exists near $D=2$, which is consistent with the present calculation results. In Refs. 14 and 15 we have discussed the reason why the analysis of Ref. 13 led to the above-mentioned result of polarization insensitivity.

We give an example of simulating a weakly fused, tapered coupler and present the coupled power variation versus draw length. The modeling procedure is the same as that described in Ref. 16, except that now the coupling structure is that of two touching cylinders. The original fibers have core diameters of 9 $\mu \mathrm{m}$ and cladding diameters of $125 \mu \mathrm{m}$. The heated region, that is, the neck section involving parallel touching cylinders, has a length of $4.3 \mathrm{~mm}$. During the drawing process, the total volume of the glass material remains constant, and the radius $a$ of the reduced cladding as well as the shapes of the two tapered regions on both sides of the heated region can be determined. The external medium is taken to be air. The power coupling can then be calculated by integration of the coupling coefficient over the entire distance over which effective coupling takes place.

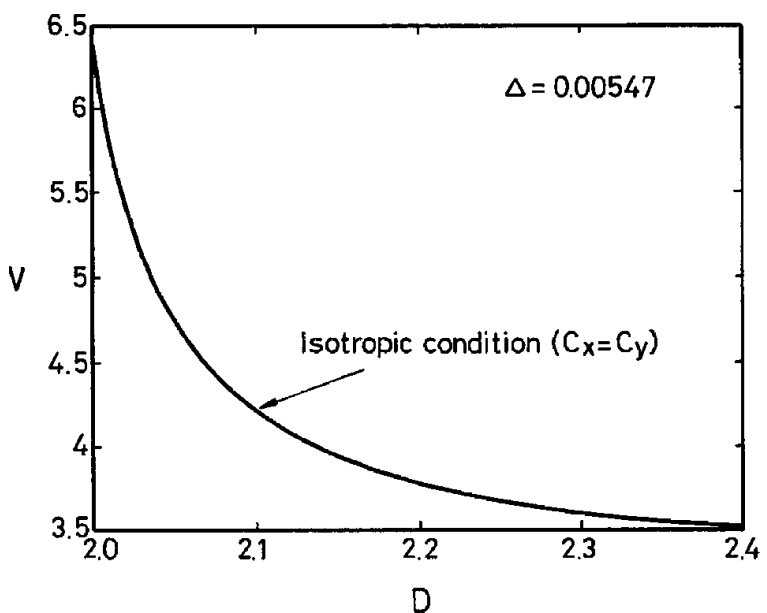

Fig. 3. Plot of the normalized frequency $V$ corresponding to $C_{x}=$ $C_{y}$ for different core separations $D$, with $\Delta=0.00547$.

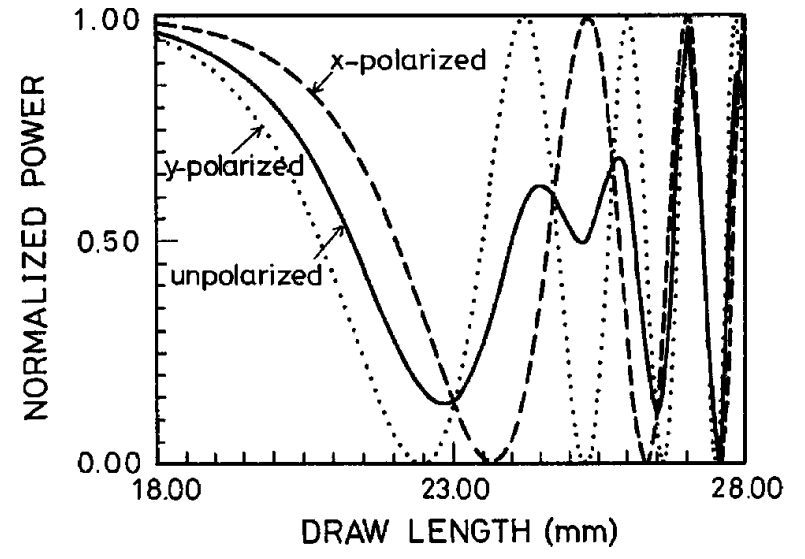

Fig. 4. Simulation results of the normalized power coupled from the input fiber to the other fiber versus the draw length for a weakly fused coupler and for three different polarization conditions.

Figure 4 gives the results in the format of normalized power coupled from the input fiber to the other fiber versus draw length, with the coupling coefficients obtained with our rigorous numerical model. When the input signal is $x$ polarized (dashed curve), the coupling is weaker than when the signal is $y$ polarized (dotted curve), consistent with Fig. 1. The solid curve in Fig. 4 shows how the coupled power behaves as the input signal is unpolarized, being simply the average of the other two curves. Because of the large difference between $C_{x}$ and $C_{y}$, the solid curve varies in a more irregular way, unlike the corresponding ones shown in Ref. 16 for the strongly fused coupler, in which a clear beating envelope appears.

In conclusion, we have calculated coupling coefficients, including the polarization effect, for the composite structure of two touching circular cylindrical fiber waveguides, in particular, for large normalized frequency values and for strongly guiding case. The calculation is based on rigorous solution of the corresponding boundary-value problem, ${ }^{11}$ and the numerical results can be treated as exact. We have compared our results in the weakly guiding case with available analytical ones 8,9 on the basis of scalar theory and have examined the accuracy of those analytical results. It is found that these scalar approximate approaches become inaccurate in predicting the polarization-splitting effect in the touchingcore situation, and the necessity of the present calculation is demonstrated. Our results should be useful in the analysis and modeling of a weakly fused tapered coupler and its polarization-beam-splitting characteristics, especially when the guiding becomes that between cladding and air.

This work was supported in part by the National Science Council of the Republic of China under grants NSC80-0417-E002-01 and NSC80-0417E002-07 and in part by the Telecommunications Laboratories, Ministry of Communications, Republic of China, under grant TL-NSC-80-5103. 


\section{References}

1. B. S. Kawasaki, K. O. Hill, and R. G. Lamont, "Biconical-taper single-mode fiber couplers," Opt. Lett. 6, 321-323 (1981).

2. V. J. Tekippe, "Passive fiber optic components made by the fused biconical taper process," in Optical Fibers and Their Applications V, R. S. Romaniuk and M. Szustakowski, eds., Proc. Soc. Photo-Opt. Instrum. Eng. 1085, 88-115 (1990).

3. T. Bricheno and V. Baker, "All-fiber polarization splitter/combiner," Electron. Lett. 21, 251-252 (1985).

4. F. P. Payne, C. D. Hussey, and M. S. Yataki, "Polarisation analysis of strongly fused and weakly fused tapered couplers," Electron. Lett. 21, 561-563 (1985).

5. M. J. F. Digonnet and H. J. Shaw, "Analysis of a tunable single mode optical fiber coupler," IEEE J. Quantum Electron. QE18, 746-754 (1982).

6. J. Bures, S. Lacroix, and J. Lapierre, "Analysis of fused single-mode optical fibre bidirectional couplers," Appl. Opt. 22, 1918-1922 (1984).

7. A. W. Snyder and J. D. Love, Optical Waveguides (Chapman \& Hall, London, 1983).

8. A. Ankiewicz, A. Altintas, A. W. Snyder, and X.-H. Zheng, "Coupling between parallel optical fiber cores-critical examination,” IEEE J. Lightwave Technol. LT-4, 1317-1323 (1986).

9. H. Renner, "Optical fiber couplers: scalar supermodes and polarization corrections," IEEE J. Lightwave Technol. 10, 728-734 (1992).

10. H.-C. Chang and T.-H. Lin, "Modeling of fused tapered optical fiber couplers through more rigorous modal analysis," in Optical Fiber Communication, Vol. 5 of 1992 OSA Technical Digest Series (Optical Society of America, Washington, D.C., 1992), p. 266.

11. H. S. Huang and H.-C. Chang, "Analysis of optical fiber directional coupling based on the $\mathrm{HE}_{11}$ modes-Part I: The identical-core case,” IEEE J. Lightwave Technol. 8, 823-831 (1990).

12. W. Wijngaard, "Guided normal modes of two parallel circular dielectric rods," J. Opt. Soc. Am. 63, 944-950 (1973).

13. X. H. Zheng, "Finite-element analysis of fused couplers," Electron. Lett. 22, 804-805 (1986).

14. T.-L. Wu and H.-C. Chang, "Rigorous analysis of form birefringence of fused fiber couplers," Electron. Lett. 30, 998-999 (1994).

15. T.-L. Wu, and H.-C. Chang, "Rigorous analysis of form birefringence of weakly fused fiber-optic couplers," IEEE J. Lightwave Technol. 13, 687-691 (1994).

16. M. Eisenmann and E. Weidel, "Single-mode fused biconical couplers for wavelength division multiplexing with channel spacing between 100 and $300 \mathrm{~nm}$," IEEE J. Lightwave Technol. 6, 113-119 (1988). 